Heine-Jahrbuch 
Herausgegeben in Verbindung mit der Heinrich-Heine-Gesellschaft 


\title{
HEINE-JAHRBUCH 2000
}

\author{
39. Jahrgang \\ Herausgegeben von Joseph A. Kruse \\ Heinrich-Heine-Institut \\ der Landeshauptstadt Düsseldorf
}

Verlag J. B. Metzler

Stuttgart - Weimar 
Anschrift des Herausgebers: Joseph A. Kruse

Heinrich-Heine-Institut

Bilker Straße I2-I4, 402I 3 Düsseldorf

Redaktion: Karin Füllner und Marianne Tilch

Die Deutsche Bibliothek - CIP-Einheitsaufnahme

Heine-Jahrbuch ... / hrsg. in Verbindung mit der Heinrich-Heine-

Gesellschaft. - Stuttgart ; Weimar : Metzler.

Erscheint jährl. - Früher im Verl. Hoffmann und Campe, Hamburg. Aufnahme nach Jg. 34. 1995

Darin aufgegangen: Heinrich-Heine-Gesellschaft: Mitteilungen der Heinrich-Heine-Gesellschaft, Düsseldorf

Jg. 34. 1995 - Verl.-Wechsel-Anzeige

ISBN 978-3-476-01790-1

ISBN 978-3-476-02722-1 (eBook)

DOI 10.1007/978-3-476-02722-1

ISSN $0073-\mathrm{I} 692$

Dieses Werk einschließlich aller seiner Teile ist urheberrechtlich geschützt. Jede Verwertung außerhalb der engen Grenzen des Urheberrechtsgesetzes ist ohne Zustimmung des Verlages unzulässig und strafbar. Das gilt insbesondere für Vervielfältigungen, Übersetzungen, Mikroverfilmungen und die Einspeicherung und Verarbeitung in elektronischen Systemen.

(C) 2000 Springer-Verlag GmbH Deutschland

Ursprünglich erschienen bei J. B. Metzlersche Verlagsbuchhandlung und Carl Ernst Poeschel Verlag GmbH in Stuttgart 2000

www.metzlerverlag.de

Info@metzlerverlag.de 


\section{Inhalt}

\section{Aufsätze}

I.

Tanja Rudtke - Die Sehnsucht nach »ewiger Kirmes « und »Mockturteltauben«. Heines »Schnabelewopski« - Eine menippeische Satire ...

Gerhard Kluge · Heines Lieblingskarikaturen. Holland, Holländisches und Holländer in den »Memoiren des Herren von Schnabelewopski«

Anne Maximiliane Jäger - Große Oper der alten neuen Welt - Überlegungen zu Heines $»$ Vitzliputzli« $\ldots \ldots \ldots \ldots \ldots \ldots \ldots \ldots$.

II.

Sikander Singh - Heinrich Heine und die deutsche Literatur des 18.

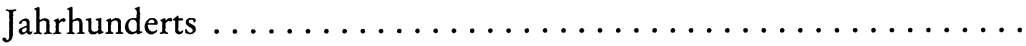

Arnold Pistiak • »Glaube, wer es geprüft«. Hölderlin - Heine: Beobachtungen. Feststellungen. Fragen . . . . . . . . . . . . . .

Thomas Schmidt-Beste $\cdot$ Felix Mendelssohn Bartholdy und Heinrich Heine

III.

Achim Hölter • »Un monument manqué«. Der Elefant auf der Place de la Bastille ............................... I 35

Gerhard Weiß - Alexander Weills »Sittengemälde aus dem elsässischen Volksleben « (I 847): volkskundliche Zeugnisse, literarische Kunstwerke und emanzipatorische Botschaften ................ 


\section{Kleinere Beiträge}

Alfredo Bauer · Die Stellung der Juden in der ersten Hälfte des 19. Jahrhunderts und Heines Übertritt zum Christentum .......... I 84

Marianne. Wirenfeldt Asmussen · Karen Blixen und Heinrich Heine .. 192 Wolfram Zöller · Das Heine-Denkmal von Carin Kreuzberg in Berlin . 200

Oh Han sin • Die neue Heine-Stele in Seoul (Hankuk-Universität). Zur Heine-Rezeption in Korea ....................... 206

\section{Berichte}

Christoph Bartscherer - Der Papst im Poetenkittel. Anmerkungen zu Heinrich Heines >blasphemischer $<$ Religiosität ............. . 209 Rudij Bergmann · Nachdenken über (m)einen Heine-Denkmal-Film .. 2 I 5

Heinrich-Heine-Institut. Sammlungen und Bestände

Bernd Kortländer $\cdot$ Neuzugänge und Erweiterungen. Nachrichten aus dem Archiv des Heine-Instituts ..................... 220

Sabine Brenner - Datenbankprojekt im Heinrich-Heine-Institut zur rheinischen Literatur und Kultur $(1871-1925) \ldots \ldots \ldots \ldots \ldots .225$

Reden zur Verleibung der Ebrengabe der Heinrich-Heine-Gesellschaft 2000

Bernhard Schlink • "Schlage die Trommel und fürchte dich nicht! « . . 230 Tilman Krause $\cdot$ Laudatio auf Bernhard Schlink ............. 238

\section{Buchbesprechungen}

Csilla Erdödy-Csorba (Hrsg.) · Europäische Romantik und nationale Identität (Ingeborg Schnelling-Reinicke) $\ldots \ldots \ldots \ldots \ldots \ldots \ldots .243$

Wilhelm Gössmann • Literatur als Lebensnerv (Gerda Kaltwasser) . . 246 
Dietmar Goltschnigg · Die Fackel ins wunde Herz. Kraus über Heine (Joseph A. Kruse) . ...............................

Peter Hasubek/ Gerd Vonhoff (Hrsg.) · Immermann-Jahrbuch (Sikan-

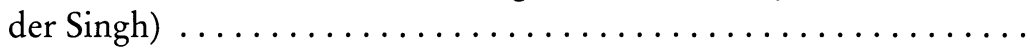

Anne Maximiliane Jäger • »Besaß auch in Spanien manch' luftiges Schloß« (Berit Balzer) ....................... 250

Roger Jones/ Martina Lauster (Hrsg.) · Karl Gutzkow (Gustav Frank) 252

Martina Lauster (Hrsg.) · Vormärzliteratur in europäischer Perspektive III (Robert Steegers) . . . . . . . . . . . . . . . . . . 254

Ralph Martin · Die Wiederkehr der Götter Griechenlands (Robert Stee-

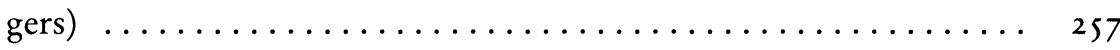

Arnold Pistiak • "Ich will das rote Sefchen küssen« (Christian Liedtke) $26 \mathrm{I}$

Irmgard Scheitler · Gattung und Geschlecht (Karin Füllner) . . . . . 263

Michael Vogt (Hrsg.) · Georg Weerth und das Feuilleton der »Neuen Rheinischen Zeitung "(François Melis) ............... 265

Martin Zimmermann · Nerval lecteur de Heine (Bernd Kortländer) . . 268

Heine-Literatur $1998 / 99$ mit Nachträgen ................ 270

Veranstaltungen des Heinrich-Heine-Instituts und der HeinrichHeine-Gesellschaft e.V. Januar bis Dezember 1999 ............ 294

Hinweise für die Autoren ......................... 300

Abbildungen ............................. 302

Mitarbeiter des Heine-Jabrbuchs 2000 ................. 303 


\section{Siglen}

I. H. Heine: Werke und Briefe

B

DHA

HSA
= Heinrich Heine: Sämtliche Schriften. Hrsg. von Klaus Briegleb. München: Hanser 1968 - 1976, 6 Bände (6, II = Register)

= Heinrich Heine: Sämtliche Werke. Düsseldorfer Ausgabe. In Verbindung mit dem Heinrich-Heine-Institut hrsg. von Manfred Windfuhr. Hamburg: Hoffmann und Campe 1973-1997

= Heinrich Heine: Werke, Briefwechsel, Lebenszeugnisse. Säkularausgabe. Hrsg. von den Nationalen Forschungs- und Gedenkstätten der klassischen deutschen Literatur in Weimar (seit I991: Stiftung Weimarer Klassik) und dem Centre National de la Recherche Scientifique in Paris. Berlin und Paris: Akademie und Editions du CNRS $1970 \mathrm{ff}$.

\section{Weitere Abkürzungen}

Galley/Estermann = Eberhard Galley und Alfred Estermann (Hrsg.): Heinrich Heines Werk im Urteil seiner Zeitgenossen. Hamburg: Hoffmann und Campe I98 Iff.

$\mathrm{HJb} \quad=$ Heine-Jahrbuch. Hrsg. vom Heinrich-Heine-Institut Düsseldorf. Hamburg: Hoffmann und Campe 1962-1994; Stuttgart: Metzler 1995 ff.

Mende $\quad=$ Fritz Mende: Heinrich Heine. Chronik seines Lebens und Werkes. Berlin: Akademie ${ }^{1}$ I $970 ;{ }^{2}$ I98 I

Seifert $\quad=$ Siegfried Seifert: Heine-Bibliographie 1954-1964. Berlin und Weimar: Aufbau 1968

Seifert/Volgina = Siegfried Seifert und Albina A. Volgina: Heine-Bibliographie 1965-1982. Berlin und Weimar: Aufbau 1986

Werner = Michael Werner (Hrsg.): Begegnungen mit Heine. Berichte der Zeitgenossen. Hamburg: Hoffmann und Campe 1973, 2 Bände

Wilamowitz = Erdmann von Wilamowitz-Moellendorf und Günther Mühlpfordt ( $\dagger$ ): Heine-Bibliographie 1983-1995. Stuttgart und Weimar: Metzler 1998

Wilhelm/Galley = Gottfried Wilhelm und Eberhard Galley: Heine-Bibliographie [bis 1953 ] . Weimar: Arion 1960, 2 Bände 